\title{
A Descriptive Study of Film Title Translation Based on a Small Self-built Parallel Corpus
}

\author{
Xuelong Jiang \\ Northwest Minzu University \\ Lanzhou, China
}

\begin{abstract}
This paper aims to observe, describe and explain the translation of film titles with the Chinese character "Xia". In this paper, a small parallel corpus is firstly constructed and annotated to analyze the basic information, language features, translation principles, strategies, methods and techniques of film titles. And then, based on Holmes' methodology of Descriptive Translation Studies, some descriptive corpus is analyzed and some assumptions are predicated. Finally, these assumptions are divided into three preliminary norms, two initial norms, two matrix norms and three textual-linguistic norms under the guidance of Toury's translation norm system with a view to provide some references and inspiration for the standardization of film title translation.
\end{abstract}

Keywords-Film title; Xia; Corpus; Descriptive Translation Study; Norms

\section{INTRODUCTION}

With internationally cultural communications becoming more and more frequent, film industry is booming and films are springing up. According to IMDB Database Statistics, 1,818,086 films were distributed from 1888 to 2016 [1]. And Hollywood Research Institute reported that global box office sales hit an all-time high of $\$ 39.92$ billion in 2017[2]. So it's no denying that film, as a vitally important medium of cultural exchanges, has already played an increasingly powerful role in our modern society.

Meanwhile, Film Title Translation (FTT) is the top priority among priorities, for film title (FT) is the soul of a film to provide the information and emotion, and it is also like an ad to improve the film promotion in the international film market, thus a good FTT can bear the persuasive function as well as the commercial function. Nevertheless, it's a pity that domestic researches of Audiovisual Translation (AVT) and FTT are relatively few and still far from satisfactory.

In China, most of FTT papers are subjective introductions of translating experience, qualitative researches of translation strategies and prescriptive studies of translation methods rather than quantitative, empirical and descriptive translation studies on the basis of corpus. What's worse, a scientific and systematic principle to guide FTT is still in lack. In 2000, Professor Qian Shaochang [3] pointed out the importance of film translation and calls on the translators to pay more attention to it. Up to now, more than seventeen years have passed, there are still many aspects of AVT and FTT to be studied, and right now it is high time for our translators to pay

This paper is supported by 2017 Fundamental Research Funds for the Central Universities (31920170050) and 2017 Scientific Research Funds for Universities in Gansu Province (2017B-86). great attention to FTT. Therefore, in light of Descriptive Translation Studies (DTS), an empirical approach and quantitative analysis are employed in this paper to study the FTT, with a view to providing some reference and inspiration for the standardization of FTT.

\section{INTERPRETATION OF DESCRIPTIVE TRANSLATION STUDIES}

\section{A. Brief Introduction of DTS}

In 1972, at the third International Conference on Applied Linguistics in Copenhagen, Holmes firstly proposed the conception of Translation Studies (TS). In light of W. Koller's translatology researching findings, Holmes defines the category of TS into three branches: Descriptive Translation Studies, Theoretical Translation Studies and Applied Translation Studies [4]. Afterwards, Even-Zohar, Toury and Maria Tymoczko make great contributions to the DTS development. Maria Tymoczko defines DTS as "the study of product, process and function under the historical background. In a broad sense, it is a translation study in connect with the politics, economy and culture" [5]. From the definition, we can conclude that DTS is an empirical science.

\section{B. The Characteristics and Methodology of DTS}

In contrast with those traditional translation theories, DTS shows some distinguished characteristics: firstly, it's an empirical science that real translation phenomenon, translating process and behavior models are studied. Secondly, it lays emphasis on the translator's subjectivity. Its aim is to study translators' translation incompleteness. Thirdly, it focuses on the target text.

Toury believes that there is a kind of comparative unit existing between the source and target texts. Actually, the comparative unit is the "coupled pair of replacing and replaced segments, which should be conceived as determining each other in a mutual way" [6]. So DTS aims to distinguish such kind of comparative unit and to illustrate that the replacing and replaced relation between the source and target texts is rational and acceptable through analysis and comparison, then further to predicate and formulate the assumptions related to such coupled pairs which will be applied to the real translation [7]. Therefore, according to Toury's theory, we sum up the DTS methodology into three steps. Step one is textual comparison and analysis; step two is to predicate the assumptions on the 
basis of comparing and analyzing results; step three is to formulate the assumptions into norms.

\section{FOUR FUNCTIONS OF FILM TITLES}

Only mastering the values and functions of FTs can the translators transmit their values as equivalent as possible to their original. So it's necessary to grasp the FT functions firstly.

It is widely known that the reason why a movie becomes a classic is not only because of its profound theme, exquisite shooting skills and actors' superb acting skills, but also owing to the significant effect of its name with profound meanings. Actually this effect is its aesthetic function which is embodied in the sound effects such as onomatopoeia, alliteration, assonance and so on and the metaphoric devices like rhythm, balance and contrasts of sentences, clauses and words. Although there are only few numbers of words in a FT, it is the essence of a film, it can reveal the film information, such as theme, plot, etc; meanwhile it is also the soul of a film, which can transmit different cultures. Furthermore, it can guide the audience's conscious orientation. It's these few words that decide the audience's choice. For commercial films, an eyecatching, gimmick title should be named so as to attract the audience, i.e., a FT is just like an ad for goods, which bears the commercial function. Above all, we can conclude that a FT basically bears informative function, cultural function, aesthetic function and commercial function.

\section{COnStruction OF A SMALl SElf-Built PARALlel CORPUS OF FILM TITLES}

We have stated above that the first step of DTS methodology is textual comparison and analysis, which is the prerequisite for the objectivity and scientificity of the translating norms. So it's required to use some effective statistic tools to analyze and compare large amount of examples. And this also accords with the aim of DTS- to observe, describe and explain the translating phenomenon. Thus, a small self-built parallel corpus of FTs with the Chinese character "Xia" is constructed, encoded and analyzed.

\section{A. Data Compilation}

The author search FTs with Chinese character "Xia" on the 1905 film internet (www.1905.com). 335 film titles are retrieved, but 239 are effective titles.

\section{B. Data Annotation}

Leech pointed out that the value of corpus can be augmented by tagging it [8]. In order to enhance the application value of parallel corpus, one of the feasible methods is to design a corpus tagging system and aggrandize its value through manual annotation [9]. Therefore, the next important work is to annotate the Basic information, language features and translation information of the corpus.

\section{1) Annotation of basic information}

Basic information includes the year of film release and type. Film types are only distinguished by Wuxia (W) and NonWuxia (N). The tagging format is "BI: year, type", so To Have and Have Not can be tagged as "BI: 1945, W".

\section{2) Annotation of language features}

Language features mainly cover character number of Chinese FT, word number of English FT, part of speech of "Xia" in the title, language structure of Chinese title and that of English title. Language structure consists of two parts: noun phrase or verb phrase; parts of speech of the phrase components. For example, “红侠” can be tagged as "n-p $(\operatorname{adj}+n)$ ", and To Kill a Rover will be marked as "v-p (v+n)". Character and word numbers are tagged like this respectively: C-number of Chinese characters, E-number of English words. Then the language feature of “女侠李飞飞 (Lee Fee-Fee the Heroine)" is tagged as "LF: C-5, E-4, n, n-p (n+n), n-p(n+n)".

\section{3) Annotation of translation information}

Translation information involves translation principle, translation strategy, translation method and translation technique.

\section{a) Translation principle}

According to FT's four functions, informative principle, cultural principle, aesthetic principle and commercial principle are proposed.

Informative principle is that translators should faithfully transfer the information of the original FT and achieve the unity of the translation title form and the original film content, i.e., to realize the equivalent of the information value. Translation is not only a process of language conversion, but also a social phenomenon of cultural exchange. Hence cultural principle implies it is one of the most important tasks to demonstrate cultural value and promote cultural exchange and understanding in FTT.

Aesthetic principle means that translators try to grasp the ideological and aesthetic content of the FT and make new artistic creation with the craftsmanship of the artist by getting rid of the shackles of the original words. The artistic beauty of the original image, emotion and language is conveyed by the artistic form of the native language, so as to transmit the aesthetic feeling to the audience. To realize the aesthetic value, we must first focus on refining the words to convey its beauty of meaning and sound.

Film is an art of both cultural and commercial nature. Commercial factors should be taken into account when translating FTs. First of all, translators should fully grasp the cultural characteristics and aesthetic taste of the target language, and create the FTs that are greatly welcomed by the target language audience, so as to arouse their psychological identification, stimulate aesthetic pleasure and generate the desire to watch. 


\section{DESCRIPTIVE ANALYSIS OF THE FILM TITLE CORPUS}

\section{b) Translation strategy}

It is a set of principles and solutions adopted in translation process to achieve specific translation goal [10]. As a macro principle, the classification of translation strategy must be closely related to the participants of translation activities. In this activity, the participants at both ends are original author and translation recipient. So according to translators' different orientations in translation activities, translation strategies can be divided into two categories: foreignization and domestication.

The essential attribute of foreignization is the original author orientation, i. e., translators try to be close to the original author in translation. In translation, the language, literature, culture and foreign flavor of the original text should be preserved as much as possible. By contrast, the essential attribute of domestication is the translation target recipient orientation, i. e., the translator tries to be close to the target recipient. In translation, translators try to replace the linguistic, literary and cultural elements of the source language with those elements that are popular with the target language readers, so as to preserve and return to the linguistic, literary and cultural norms of the target language.

\section{c) Translation method}

Based on translation strategy, FTT methods can be divided into foreignizating translation methods including zero translation, transliteration, interlinear translation and literal translation and domesticating translation methods including liberal translation, borrowing translation, imitative translation, adaptation and creative translation.

\section{d) Translation technique}

It includes amplification, omission and conversion. And conversion is further classified as lexical conversion (conversion of parts of speech), semantic conversion (conversions of expressing patterns, specific and general), syntactic conversion (word order conversion) and cultural conversion (cultural image conversion).

So marks of translation information are as follows:

TP: i-p, cul-p, a-p, com-p; TS: f, d; TM: zero trans, transliteration, interlinear trans, literal trans, liberal trans, adaptation, creative trans; TT: ampli, omis, convers; Lexical conversion: POS (n-v), POS (v-n), POS (n-adj), POS (v-adj); Semantic conversion: expressing pattern, specific-general conversion; Syntactic conversion: word order; Cultural conversion: cul-image.

\section{“黑衣女侠(The Heroine in Black)” can be tagged as:}

黑衣女侠(The Heroine in Black) <BI: 1928, W >; <LF: C-4, E-4, n, n-p(adj+adj+n), n-p(n+prep) >; $\langle$ TP: i-p $\rangle$; $\langle$ TS: f $\rangle$; <TM: literal trans>; <TT: cul-image>

\section{A. Descriptive Analysis of Basic Information}

Corpus capacity is 239 FTs with "Xia" from 1924 to 2016. The earliest film is A Young Gallant Man of 1924, and 22 films are between 1920 and 1929, 10 between 1930 and 1939, 13 from 1940 to 1949,27 from 1950 to 1959,57 from 1960 to 1969, 23 from 1970 to 1979,23 from 1980 to 1989,28 from 1990 to 1999,16 from 2000 to 2009, 20 from 2010 to 2016. Through the decade distribution scale, we can find there is an obvious climax between 1960 and 1969, which is consistent with the peak of Wuxia novels in China. It is well-known that during fifties and sixties, Chinese Wuxia novelists such as Liang Yusheng, Jin Yong and Gu Long wrote many famous novels. Meanwhile, the articulation from 1930 to 1939 is the lowest due to WWII.

As to film types, 18 of 239 films are non-Wuxia films, among which cartoon (5 films), science fiction (3 films) and modern action movie (10 films) are involved.

\section{B. Descriptive Analysis of Language Features}

In terms of the word number of titles, four-character titles are the majority in Chinese versions, accounting for $41 \%$, while in English versions, the majority are three-word titles with the percentage of $29 \%$ and four-word titles with proportion of $23 \%$. The reason may be the English form of "article+adjective+noun" is usually correspond to the Chinese four-character phrase. Among 97 Chinese four-character titles, the number of 2-4 words English versions is 67, the number of one word is 2 and the number of 7 words is 2 . The title with the most Chinese characters is “女飞侠黄芶巧破钻石党” with 11 characters; while that of the most English words is How the Woman Security Escort Thrice Fought the Hero with the Magnificent Whip with 13 words. The least number of characters in Chinese is 2, and the least of English version is only one.

Chinese character "Xia" is often used as a noun, nevertheless in FTs it can also be used as an adjective. In the FT corpus, 198 are with "Xia" as a noun, 41 are used as adjectives. In English versions, the adjectives corresponding to "Xia" are "gallant", "magnificent" and "chivalrous" etc.

As to language structure, Chinese FTs with noun phrase structure are 209, the amount of titles with SVO structure is 15, and the rest are with verb phrase structure. In the $n-p$ titles, the majority are $n-p(n+n)$ and $n-p(\operatorname{adj}+n)$ structures. The $n-p(n+n)$ structure is mainly appositive structure, like “大侠张汶祥”. By comparing Chinese appositive structure with the English one, we can find the Chinese structure is "Xia+person's name", while the English structure is "Person's name+Xia", which is due to different language codes. Language structures of English FTs are more diversified, the number of titles with noun phrase structure is 214 , that with verb phrase structure is 8 , that with adjective phrase structure is 4 , and the amount of sentence titles is 11 . The sentence structures consist of SVO sentence, SV sentence, inverted sentence, How special question and Why special question. By comparing, we can find that titles of Chinese SVO sentence structure are mostly transferred into English special questions. 


\section{Descriptive Analysis of Translation Information}

1) Descriptive analysis of translation principle:

As to translation principles, 199 of 239 Chinese FTs are translated into English versions with priority of the informative principle. 33 film titles are translated under the guidance of cultural principle, for instance: “蛮女侠” are translated into Temperamental Amazon. In Greek mythology amazon was a tribe of women warriors related to Scythians and Sarmatians. So this English FT reflects western culture. Meanwhile, this FT is also guided by the informative principle, as the content of the original FT is faithfully conveyed in the English version. There are also translations guided by both cultural and commercial principles, such as the translation of “侠胆雄狮” into Beauty and the Beast which is so popular in western culture that the title can not only transmit the cultural information but also arouse audiences' psychological identification and generate their desire to watch. FTTs directed by aesthetic principle are very few, only three titles are found, such as “风尘三女侠 (Why Wild Girls)" which has a rhythmic beauty with the usage of alliteration.

\section{2) Descriptive analysis of translation strategy:}

In western culture, there doesn't exist the spirit of Xia and there is no equivalent to Chinese Xia in English, so there is a cultural gap. Therefore, the Xia in 64 titles are paraphrased or omitted; 174 titles are translated by employing domesticating translation strategy, 45 different words familiar to target recipients are used. Only one title is adopted foreignizating translation strategy, it is “仙侠剑 (Xian-Xia Sword)”. The figure of the 45 words is as follows:

- hero/heroine
q girl/boy
- lover
m Mr./Mrs.
- tiger
conman
- he/she
- lad
m maverick

- swordman
- Musketeer
amazon
ninja
m trio
fool
- heroic
lady
a prince

$$
\begin{aligned}
& \text { man/woman } \\
& \text { - chivalrous } \\
& \text { duo } \\
& \text { valeface } \\
& \text { - assassin } \\
& \text { gallantry } \\
& \text { - him } \\
& \text { - loner } \\
& \text { rover }
\end{aligned}
$$

knight
chivalry
mighter
ranger
m boxer
ghost
giller
magician
m the young

$$
\begin{aligned}
& \text { - warrior/warrioress } \\
& \text { - king } \\
& \text { master } \\
& \text { - savior } \\
& \text { child } \\
& \text { - God } \\
& \text { - kungfu } \\
& \text { maiden } \\
& \text { mamp }
\end{aligned}
$$

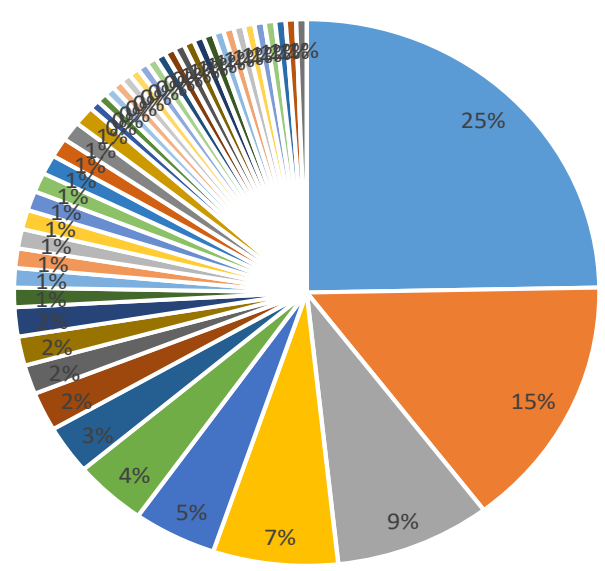

Fig. 1. English words translated from Chinese "Xia" in film titles

From the above chart, we can find "hero, swordsman, man, knight and warrior" are the five highest-frequency words respond to Xia. In the five words, "hero" has some similarity with Xia, the meanings of swordsman, knight, warrior, assassin, ranger, musketeer and savior distinguish from that of Xia. According to Kangxi Dictionary, Xia refers to the person who has the ability to help those who are weaker than themselves without seeking return. This is also a kind of spirit and social pursuit in ancient China. However, swordsman refers to someone skilled at fencing, but Chinese Xia is not only good at fencing but also keeping the faith of drawing swords at the sight of injustice; knight indicates a person of noble birth trained to arms and chivalry, but Xia isn't necessarily highborn; warrior refers to someone engaged in or experienced in warfare, but Xia isn't necessarily a soldier; assassin refers to a murderer who kills by a treacherous surprise attack and often is hired to do the deed, but Xia helps others for morality rather than for money; ranger formerly is used to refer to a mounted lawman who maintained order on the frontier, but Xia isn't an officer; musketeer is a foot soldier armed with a musket, but Xia generally is not a soldier and uses swords instead of guns; savior is involved with Christianity, but Xia has no relation with religion. In conclusion, the conception of Chinese $\mathrm{Xia}$ is much broader and more obscure than those of knight, assassin, ranger, musketeer, savior, etc. 
We can also find that in English the words with broader meaning like "man/woman, boy/girl, lad, lady, child" are also used to paraphrase Xia. They have a common feature which is that adjectives as "gallant, chivalrous, kungfu, etc" are used to modify those nouns, for examples, The Gallant Girl Nicknamed White Rose and Brave Lad of Guangdong. There are two exception: Man of Tai Chi and Man Hunter. In the latter title, "man" functions as an adjective. And in 239 Chinese titles, "Xia" in 41 titles are used as adjectives, but in English versions, only 7 titles are translated "Xia" as adjectives which are "gallant, chivalrous (2 times), heroic, great, noble and loyal".

\section{3) Descriptive analysis of translation method:}

As to translation methods of film titles, there is a table in the following.

TABLE I. TABLE OF TRANSLATION METHODS

\begin{tabular}{|c|c|c|}
\hline Translation Method & Title Amount & Proportion (\%) \\
\hline literal translation & 104 & 43.5 \\
\hline liberal translation & 64 & 26.8 \\
\hline adaptation & 35 & 14.6 \\
\hline Transliteration & 12 & 5.0 \\
\hline interlinear translation & 11 & 4.6 \\
\hline creative translation & 6 & 2.5 \\
\hline borrowing translation & 4 & 1.7 \\
\hline imitative translation & 3 & 1.3 \\
\hline
\end{tabular}

Form the above table, we can know that the three most frequent used translating methods are literal translation, liberal translation and adaptation. Transliteration, creative, borrowing and imitative methods are employed less frequent. Transliteration is mainly used in the titles with person's names. And creative translation is only used six times, borrowing and imitative methods are even less. “江湖情侠” is creatively translated into $\mathrm{He}$ and She, which expresses not only the information that leading characters of the film are a couple, but also presents a kind of concision aesthetics. “侠胆雄狮” is rendered into Beauty and the Beast which is a very popular story for westerners. And “大灭侠” is translated into To Kill a Rover by imitating Harper Lee's famous novel To Kill a Mockingbird.

\section{4) Descriptive analysis of translation technique:}

TABLE II. TABLE OF TRANSLATION TECHNIQUE

\begin{tabular}{|c|c|c|c|c|c|}
\hline \multicolumn{2}{|c|}{ Translation Technique } & \multicolumn{2}{|c|}{ Title Amount } & \multicolumn{2}{|c|}{ Proportion (\%) } \\
\hline \multicolumn{2}{|c|}{ amplification } & \multicolumn{2}{|c|}{11} & \multicolumn{2}{|c|}{4.6} \\
\hline \multicolumn{2}{|c|}{ omission } & \multicolumn{2}{|c|}{40} & \multicolumn{2}{|c|}{16.7} \\
\hline \multirow{4}{*}{ conversion } & lexical & \multirow{4}{*}{221} & 8 & \multirow{4}{*}{92.5} & 3.6 \\
\hline & semantic & & 113 & & 51.1 \\
\hline & syntactic & & 3 & & 1.4 \\
\hline & cultural & & 118 & & 53.4 \\
\hline
\end{tabular}

a) Conversion: It is the most frequently used technique, which is subdivided into lexical, semantic, syntactic and cultural levels. Firstly, lexical conversion is mainly about shift of parts of speech, specifically including shift from noun to verb (1 title), from verb to noun (2 titles), form noun to adjective (3 titles) and from verb to adjective (2 titles). “旋风 双侠” is the example of shift from noun to verb in a loose sense. In this title, “旋风(cyclone)" is a noun which is converted into a verb in its English translation Saddle the Wind. From this translation, we can infer that the translator employed interlinear translation, and he considered “旋” as a verb and “风” as the object of the verb, which is not correct. “飞剑侠血 战周家庄(The flying swordsman's bloody battle at Zhou's Manor)” and “峨嵋飞剑侠误闯风火岛 (The Flying Swordsman's Adventure on the Island of Wind and Fire)" are the two examples of shift from verb to noun, for two verbs "血 战” and “误闯” are converted into two nouns "battle” and "adventure" respectively. And there are three examples of shift from noun to adjective: “万侠之王(The Chief Warrior)”, “雌 雄双侠(The Chivalrous Pair)” and “东方三侠(The Heroic Trio)”. “复仇” in “大侠复仇记(A Revengeful Man) and “留 香” in “侠影留香(Everlasting Chivalry)” are converted into adjectives "revengeful" and "everlasting".

Secondly, semantic conversion can be further classified into shift of expressing pattern (59 titles), specific-general shift (16 titles) and general-specific shift (38 titles). Expressing pattern shift is subdivided into two categories, one is noncorresponding shift (48 titles), the other is sentence pattern shift (11 titles). “王氏四侠(Fury in Their Hearts)” can be cited to illustrate the non-corresponding shift. The Chinese FT means four heroes surnamed Wang, but its corresponding English translation is Fury in Their Hearts, from which we can find no semantic relation with its original title. The Chinese titles employed sentence pattern shift are all very long and declarative sentences, like “关东小侠大破莲花阵” and “峨嵋 飞剑侠横扫金银宫”. In English versions, they're both shifted into special questions How the Young Northeast Hero Shattered the Lotus Militia and How the Flying Swordsmen from Mount Emei raided Gold and Silver Palace. This shift of sentence pattern can stimulate audiences' curiosity, which is beneficial to the commercial value of film titles. As to specificgeneral shift, it implies that the specific Chinese cultural image $\mathrm{Xia}$ is transferred into relatively general English words like "man/woman, boy/girl, lad, lady, child". On the contrary, general-specific shift indicates that the Chinese $\mathrm{Xia}$ is converted into specific English cultural images like "hero, swordsman, man, knight, warrior, etc". The examples of the two shifts have been explained in the above part of domesticating translation strategy.

Thirdly, syntactic conversion actually refers to shift of words order, with only three such examples of shifting form the order "Xia+person's name" to the order of "Person's name+Xia". And fourthly, cultural conversion is the shift of cultural images, namely, the shift from Chinese Xia into English heroes, swordsmen, men, knights, warriors, etc, which are overlapped with general-specific and specific-general shifts. 
b) Omission: It is the second most frequently used translation technique, which is employed in 40 titles. Some person's names in titles are omitted, such as “女侠文婷玉(The Lady Musketeer)"; some weapons are deleted, such as in “神笛 正侠 (The Begging Swordsman)” “神笛 (magic flute)” is deleted; some Chinese cultural words are omitted, for example, in “乾坤大醉侠(The Drinking Knight)”, “乾坤(hexagrams of Qian and Kun)" is omitted; and some verbs are deleted, like “歼仇(kill enemies)” in “虎侠歼仇(Tiger Boy)” is deleted.

c) Amplification: The least employed technique is amplification, with only 11 examples. Nouns and adjectives are added. For instances, in “独行侠(The Legend of the Lone Ranger)", "the legend" is added; and the adjective "deadly" in “双侠(The Deadly Duo)" is added.

Finally, an important point need to notice is that 113 titles are translated by using more than one translation techniques, that's why the sum of titles employed amplification, omission and conversion isn't 239 and the sum of titles used lexical, semantic, syntactic and cultural conversions is 242 that is bigger than the totality of titles employed conversion 221.

5) Descriptive analysis of high-frequency phrases and words except "Xia"

a) Translation of numbers in film titles: In the corpus, There are 44 film titles that contains numbers "one, double, three, three and a half, four, five, seven, eight, thirteen, sixteen, nineteen, twenty-four, hundred, ten thousand". After translating into English, only seven titles retain the original numbers, for examples of “江湖二十四侠(24 Heroes)” and “青城十九侠 (19 Heroes of the Green Mountain)". Numbers of three titles are transformed into nouns with numeral implication, such as “边城三侠 (The Magnificent Trio)”, “雌雄双侠 (The Chivalrous Pair)" and “双侠(The Deadly Duo)". Numbers are deleted in the rest of titles, like “七侠五义(King Cat)".

b) Translation of four-character idioms: There are 98 Chinese titles containing four-character idioms in the corpus. As a treasure of the Chinese language and culture, fourcharacter idiom is characterized as symmetry, conciseness and vividness. While English is a morphological language, which uses formal meaning to represent grammatical categories and semantic information with strict morphological changes. Hence, most of the four-character idioms are combined and adapted. “妖光侠影” and “侠骨仁心” are combined into Evil's Shadow and Healing Hearts, and “侠影 (the shadow of hero)" and “侠骨 (chivalrous disposition)" are deleted, because they are coordinate structures. The second method to translate Chinese four-character idioms is adaptation. 58 titles are adapted and the structures of four-character are abandoned, just like“侠凤奇缘” into Reciprocity and “侠胆双雄” into Backfire.

c) Translation of appositive structures: Among 34 appositive Chinese film titles, only 9 English titles keep the structure including the usage of dash in one title “北侠欧阳春 (Northern Knight-errant -Chun Oyang) ". And there is also one title transferred into a special question sentence “飞侠吕三 娘(How Lu Shanniang Flies in the Air)". And the rest all just maintain the Xia's names or nicknames like “女侠黑蝴蝶
(Black Butterfly)", which is in accordance with the film title naming rules- naming after the protagonist.

d) Translation of Chinese traditional weapons: In the 239 film titles, there are 29 titles with Chinese traditional weapons blade, sword, spear, fishgig, whip and flute. Swords and whips of the 29 titles are retained in the English versions, and the rest weapons are all omitted, maybe because those Chinese weapons are not familiar to the foreigners, keeping them in the title may make westerners puzzled.

e) Translation of category words: In Chinese FTs, category words like “传(biography), 记(diary), 录(record), 行 (log) and 传奇(legend)" are frequent used. Biography is a kind of Chinese stylistic format, which consists of two categories, one is historical biography based on historical facts, and the other is literary biography in light of history but including some imaginative descriptions. And the latter is similar to legend. In the 239 titles, 11 ones are with "biography, diary, record, log and legend", of which three titles maintain these words in translations, they are "醉侠行(Legend of Drunken Tiger)”, “兔侠传奇(Legend of a Rabbit) and “侠客行(Ode to Gallantry)". The rest are all deleted in English titles like “侠义 英雄传(The Queen and the Magician)”, “原野奇侠传(Revenge of a Swordsman)”, “豪侠传(Killers Five)”, “隐侠恩仇录(The Mysterious Heroes)", etc.

\section{CONCLUSIONS}

The term "norm" was firstly proposed by Gideon Toury, which refers to both regularity in behaviour, i.e. a recurring pattern, and to the underlying mechanism which accounts for this regularity. The mechanism is a psychological and social entity, which mediates between the individual's intentions, choices and actions, and collectively held beliefs, values and preferences... According to Toury, in the process of translation, translators are restricted by three categories of norms: preliminary norms, initial norms and operational norms. The first category of norms mainly involves the choices of source texts and translating decisions starting from the source texts; the second one refers to the general tendency of translation, which restricts the choices of two extreme tendencies: adequacy and acceptability. Adequacy refers to the tendency of closing to the source language and culture; while acceptability refers to the tendency of closing to the target language and culture. Operational norms are the decisions making in the translation activity, which includes matrix norms and textuallinguistic norms. The former involve the macroscopic level like the arrangement and acceptance or rejection of textual contents the latter involve the microcosmic level like sentence structure, wording and phrasing [11].

So in accordance with Toury's theoretical frame of norms, and on the basis of the above descriptive analysis of FTT, three preliminary norms, two initial norms, two matrix norms and three textual-linguistic norms are formulated.

Preliminary Norms One: the number of words in a film title is limited within 8 words, and Chinese film titles are suggested to employ the four-character structure as frequent as possible. Noun phrase structure is the commonest structure in film titles, sentence structures are suggested to use as few as possible. 
Preliminary Norm Two: film titles generally are named by three rules: 1) naming after plot or theme; 2) naming after protagonist; 3) naming after time, place or background.

Preliminary Norm Three: a film title basically bears four functions: informative function, cultural function, aesthetic function and commercial function.

Initial Norm One: FTT methods are divided into two groups: foreignizating translation methods (including zero translation, transliteration, interlinear translation and literal translation) and domesticating translation methods (including liberal translation, borrowing translation, imitative translation, adaptation and creative translation).

Initial Norm Two: in FTT, literal translation and adaptation are the most frequently used methods, and conversion is the most frequently used technique.

Matrix Norm One: four translation principles are summarized to guide FTT: informative principle, cultural principle, aesthetic principle and commercial principle. The four FTT principles are an entity.

Matrix Norm Two: the criteria of evaluating a FTT is whether the four principles are all taken into account.

Textual-linguistic Norm One: FTT techniques include amplification, omission, and conversion. And conversion is further classified as lexical conversion, semantic conversion, syntactic conversion and cultural conversion.

Textual-linguistic Norm Two: three methods are proposed to translate Chinese character Xia in film titles: 1) paraphrasing by using different words familiar to target recipients; 2) transliterating; 3) omitting.

Textual-linguistic Norm Three: three methods to translate appositive structure in the FFT can be summarized: retaining both of the appositive constituents, retaining both of the appositive constituents by using a dash, and retaining either of the appositive constituents.

\section{REFERENCES}

[1] IMDB. Worldwide Films Database [DB/OL]. (2017-03-01) [2018-0723]. www.imdb.com.

[2] Data Center of Hollywood Institute. Global Film Industry Research Report of 2017 [R/OL]. (2018-02-07) [2018-07-23]. https://zhuanlan.zhihu.com/p/33655648.

[3] Qian Shaochang. "Film Translation- a More and More Important Field of Translation Study" (In Chinese), Chinese Translators Journal, vol. 1, pp. 61-65, February 2000.

[4] Toury, Gideon. Descriptive Translation Studies and Beyond. Philadelphia: The John Benjamins Publishing, 1995, pp. 10-18.

[5] Lin Kenan. "Translation Studies: from Prescription to Description" (In Chinese), Chinese Translators Journal, vol. 6, pp. 43-45, December 2001.

[6] Toury, Gideon. A Handful of Paragraphs on "Translation" and "Norms" [A]. Ed. C. Sehafther. Translation and Norms. Clevedon: Multilingual Matters Ltd, 1999, pp. 80-81.

[7] Liu Tengteng. A Descriptive Study of Chinese Translation of English Film Titles: A Corpus-Based Approach [A]. Changsha: Central South University, pp. 29, November 2011.

[8] Leech G. Introducing Corpus Annotation [A].In Garside R, Leech G \& McEnery A (eds.). Corpus Annotation: Linguistic Information from Computer Text Corpora [C]. London: Longman, 1997, pp. 1-18.

[9] Wanghui, Zhu Chunshen. "The Annotation and Application of Translation Teaching Corpus- Introduction of the Corpus of Chinese Translation and Notes in English Financial Reports" (In Chinese), Foreign Language Teaching and Research, vol. 2, pp. 246-255, April 2000.

[10] Xiongbing. "Study of Translation Teaching Model on the Basis of English-Chinese Parallel Corpus" (In Chinese), Foreign Language World, vol. 169, pp. 2-10, August 2015.

[11] Hermans, Theo. Translation in Systems: Descriptive \& System-oriented Approaches Explained. Manchester: St. Jerome Publishing, 1999, p. 80. 